\title{
A Review of the Source and Function of Microbiota in Breast Milk
}

\author{
M. Susan LaTuga, MD, MSPH ${ }^{1} \quad$ Alison Stuebe, MD, MSc ${ }^{2,3}$ Patrick C. Seed, MD, $\mathrm{PhD}^{4}$ \\ ${ }^{1}$ Department of Pediatrics, Division of Neonatology, Albert Einstein \\ College of Medicine, Bronx, New York \\ 2 Department of Obstetrics and Gynecology, University of North \\ Carolina School of Medicine \\ ${ }^{3}$ Department of Maternal and Child Health, Gillings School of Global \\ Public Health, Chapel Hill, North Carolina \\ ${ }^{4}$ Department of Pediatrics, Division of Infectious Diseases, Duke \\ University, Durham, North Carolina \\ Semin Reprod Med 2014;32:68-73 \\ Address for correspondence M. Susan LaTuga, MD, MSPH, Albert \\ Einstein College of Medicine, 1601 Tenbroeck Ave, 2nd floor, Bronx, NY \\ 10461 (e-mail: mlatuga@montefiore.org).
}

\begin{abstract}
\section{Keywords}

- enteromammary trafficking

- immune function

- mastitis

Breast milk contains a rich microbiota composed of viable skin and non-skin bacteria. The extent of the breast milk microbiota diversity has been revealed through new culture-independent studies using microbial DNA signatures. However, the extent to which the breast milk microbiota are transferred from mother to infant and the function of these breast milk microbiota for the infant are only partially understood. Here, we appraise hypotheses regarding the formation of breast milk microbiota, including retrograde infant-to-mother transfer and enteromammary trafficking, and we review current knowledge of mechanisms determining the extent of breast milk microbiota transfer from mother to infant. We highlight known functions of constituents in the breast milk microbiota-to enhance immunity, liberate nutrients, synergize with breast milk oligosaccharides to enhance intestinal barrier function, and strengthen a functional gut-brain axis. We also consider the pathophysiology of maternal mastitis with respect to a dysbiosis or abnormal shift in the breast milk microbiota. In conclusion, through a complex, highly evolved process in the early stages of discovery, mothers transfer the breast milk microbiota to their infants to impact infant growth and development.
\end{abstract}

Breast milk provides optimal nutrition for infants and reduces their risk of infectious diseases. In addition, breast milk is a vehicle for transmission of bacteria and viruses from mother to infant. However, the factors dictating the composition of the breast milk microbiota and the function of the breast milk microbiota for the mammary organ and infant remain unclear. Breast milk typically contains both skin microbiota and what are typically considered enteric organisms. Proposed theories for the microbiota composition of breast milk include retrograde flow from the infant's oral cavity, transfer of organisms from maternal skin, and movement of microbiota from the maternal enteric tract to the mammary gland. Here, we review mechanisms for transfer of microbiota to breast milk, their potential function for infants, as well as dysbiosis of the breast milk microbiome related to maternal disease.
Until recently, our knowledge of the ecology of humanassociated microbes was based primarily on bacterial culture. However, culture-based methods may be limited by our ability to optimize growth conditions. As such, fastidious and low-abundance organisms may not be identified using culture methods. As many as $60 \%$ of organisms detected using molecular techniques will not grow in standard bacterial culture media. ${ }^{1,2}$ While detection using molecular techniques has broadened our understanding of microbiota, many sequences cannot be classified, suggesting an expansive microbial world.

Within the human body, microbial communities assemble that are specific to location but not isolated from one another. $^{3}$ For example, skin-associated microbiota are rich in gram-positive organisms such as Staphylococcus spp. and
Issue Theme The Microbiome and Reproduction; Guest Editors, James H. Segars, MD, and Kjersti M. Aagaard, MD, PhD
Copyright @ 2014 by Thieme Medical Publishers, Inc., 333 Seventh Avenue, New York, NY 10001, USA. Tel: +1(212) 584-4662.
DOI http://dx.doi.org/ 10.1055/s-0033-1361824. ISSN 1526-8004. 
Propionibacterium spp. ${ }^{4,5}$ In comparison, the adult enteric microbiota are more diverse and generally dominated by anaerobic organisms such as Bacteroides and Prevotella. ${ }^{6}$ Using culture and molecular techniques, breast milk contains organisms typically identified as both skin- and entericassociated organisms such as Staphylococcus, Streptococcus, Escherichia, Enterococcus, Veillonella, Prevotella, Pseudomonas, and Clostridia ${ }^{7-12}$ (-Table 1). Several associated factors influence the composition of the breast milk microbiota, including maternal health and mode of delivery (-Table 2 ). Furthermore, the composition is dynamic, changing from high diversity including typical skin- and enteric-type organisms in colostrum to less diverse flora with greater infant oral and skin microbiota as lactation progresses (-Fig. 1).

Models that are not mutually exclusive have been proposed for how breast milk contains viable diverse microbiota. Microbiota typically associated with the skin may be transferred to breast milk. Molecular approaches have been employed to genetically type gram-positive organisms from the maternal skin, breast milk, and her infant to demonstrate the commonality of specific strains in the dyad, thus suggesting that skin bacteria may be transferred in breast milk or through the process of breastfeeding from mother to infant. $^{13,14}$ In studies examining microbiota in breast milk, before expressing milk for examination, mothers were instructed to perform special cleansing of the breast skin surface. ${ }^{10-12}$ Even after cleaning the periareolar area, these breast milk samples contain viable skin- and enteric-associated microbiota. In practice, mothers do not cleanse their breast before breastfeeding. In addition, during breastfeeding, the nipple and surrounding areolar region are in the infant's mouth introducing maternal skin-associated bacteria to the infant's oral cavity and enteric tract. ${ }^{15}$

Others have proposed that organisms travel in a retrograde fashion from the infant's oral cavity into the ductal tissue. ${ }^{10}$ Based on the physiology of infant suckling, there may be backward flow of breast milk from the infant's oral cavity through the nipple into the mammary gland. ${ }^{16,17}$ This mechanism may explain the presence of organisms that have been noted in the oral cavity of both neonates and breast milk such as Gemella, Veillonella, Staphylococcus, and Streptococcus. ${ }^{10,18}$ However, other organisms typically noted in the oral cavity of

Table 1 Bacteria commonly found in breast milk using culture and molecular techniques

\begin{tabular}{|l|l|}
\hline Phyla & Genera \\
\hline Firmicutes & $\begin{array}{l}\text { Staphylococcus, Streptococcus, Veillonella, } \\
\text { Gemella, Enterococcus, Clostridia, } \\
\text { Bifidobacterium, Lactobacillus }\end{array}$ \\
\hline Actinobacteria & $\begin{array}{l}\text { Propionibacterium, Actinomyces, } \\
\text { Corynebacterium }\end{array}$ \\
\hline Proteobacteria & $\begin{array}{l}\text { Pseudomonas, Sphingomonas, Serratia, } \\
\text { Escherichia, Enterobacter, Ralstonia, } \\
\text { Bradyrhizobium }\end{array}$ \\
\hline Bacteroidetes & Prevotella \\
\hline
\end{tabular}

Source: Data adapted from Hunt et al ${ }^{10}$; Thompson et al ${ }^{12}$; Perez et al ${ }^{14}$; Cabrera-Rubio et al ${ }^{11}$; and Delgado et al. ${ }^{61}$
Table 2 Factors influencing breast milk microbiota community structure

\begin{tabular}{|l|l|}
\hline Maternal factors & Postnatal factors \\
\hline Obesity & Mode of delivery \\
Atopy & Gestational age \\
Diet & Maternal antibiotic use \\
Immunologic status & Stage of lactation \\
\hline
\end{tabular}

neonates such as Actinomyces have not been consistently found in breast milk. In addition, initial colostrum samples contain DNA signatures of bacteria before infants have breastfed. ${ }^{11}$ Thus, though milk transfer from an infant's oral cavity may explain the presence of some organisms, it does not fully explain the composition of the breast milk microbiota.

Recently, an alternative model has been proposed to account for the presence of typically enteric organisms in breast milk. Evidence suggests that mucosal intestinal dendritic cells regularly engulf intestinal bacteria, which may subsequently be trafficked into the systemic circulation. ${ }^{19}$ In pregnant and lactating women, these leukocytes with intracellular bacteria may be trafficked to the mammary gland and secreted into breast milk. These organisms or parts of organism may directly seed the infant enteric tract or alter the community structure, providing the basis for a model of enteromammary trafficking (EMT).

In support of the EMT model, in three studies of mothers and their term infants, a subset of genomic signatures corresponding to Bifidobacterium longum, Streptococcus thermophilus, and Bifidobacterium pseudocatenulatum were common to maternal stool, maternal blood, breast milk, and infant stool samples. ${ }^{14,20,21}$ In terms of viruses, human immunodeficiency virus (HIV) in breast milk appears to be distinct based on phylogenetic classification from HIV found in the peripheral blood of infected mothers. ${ }^{22}$ Some have proposed that the strains of HIV found in breast milk may originate from gut-associated lymph tissue, with subsequent trafficking via infected lymphocytes to the mammary gland. ${ }^{23}$ Because this process is dependent on maternal enteric microbiota, it may be affected by maternal diet, body habitus, immunological status, and geography. 6,24

Another potential mechanism for transfer of microbiota from mothers into breast milk involves spread from the mammary gland. In a murine animal model of cytomegalovirus (CMV), virus may remain quiescent in the mammary gland following primary infection. ${ }^{25}$ The process of lactation is proposed to reactivate these viruses. Consistent with this

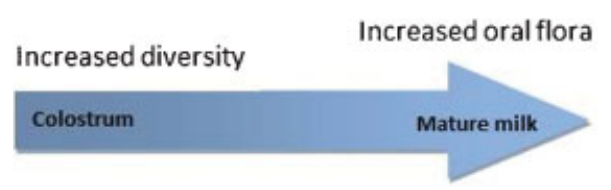

Figure 1 During the course of lactation, there is an overall decrease in bacterial diversity compared with colostrum samples. The composition of the microbiota shifts from skin- and enteric-associated organisms to infant oral and skin organisms. 
idea, CMV has been detected in the breast milk of asymptomatic CMV seropositive women. Virolactia, the presence of live virus in breast milk, correlates with duration of lactation and peaks during weeks 3 to 4 of lactation. In addition, the shedding of CMV appears to be limited to breast milk. ${ }^{26}$ Premature infants may be at risk of postnatal acquisition of CMV from breast milk due to decreased transplacental transmission of antibodies against CMV. ${ }^{27}$ Because of the benefits of breast milk for premature infants, in most neonatal intensive care units, breast milk is frozen before administration, decreasing the inadvertent transmission of CMV via breast milk. ${ }^{28}$ Recent data suggest the CMV shedding in mothers of premature infants, also may be influenced by local immune factors in the mammary gland. ${ }^{29}$

\section{Proposed Function of Microbiota in Human Breast Milk}

The function of microbiota in breast milk may include enhanced immune development through microbial ligands, nutrient metabolism and absorption, improved intestinal barrier function, and stimulation of the gut-brain axis. All neonates have immature immune function, as evidenced by increased anti-inflammatory $\mathrm{T}$ regulatory cell populations in cord blood. ${ }^{30}$ Within the CD4 positive T-cell population, there are T helper 2 (Th2) and T helper 1 (Th1) cells. Th1 cells produce interleukin (IL) 2, interferon, and tumor necrosis factor, all of which promote cytotoxic T-cell function. In comparison, Th2 cells secrete IL4, IL5, IL6, IL10, and IL21, which support humoral immunity. ${ }^{31}$ Infants have higher Th2: Th1 ratios compared with adults, suggesting an enhanced B-cell response and potential for allergic sensitization. ${ }^{32}$

Feeding with human milk advances immune development in full-term infants: compared with formula-fed infants, breastfed infants demonstrate increased Th1 activity, higher proliferative T-cell response to tetanus toxoid, ${ }^{33}$ and moderated CD4 counts using flow cytometry. ${ }^{34}$ In a small study of preterm infants, infants fed breast milk also had lower B-cell counts than infants who were fed formula. ${ }^{35}$ Because of the immaturity of the neonatal humoral response, infants facing infection rely on maternal antibodies and a robust cytotoxic Th1 response. Enhanced cytotoxic function in infants fed breast milk may be promoted by bacterial ligands in breast milk. ${ }^{36}$ In support of this theory, in vitro stimulation of dendritic cells with lipopolysaccharide supported T-cell differentiation. ${ }^{37}$ Animals raised in a germ-free (GF) environment had lasting impairments in their immunologic function. ${ }^{38}$ The effect of breast milk on maturation of Th17 cells remains unclear. ${ }^{39}$ Thus, microbiota in breast milk may stimulate maturation of cytotoxic Th1 cells and improve their ability to fight infection.

In the enteric tract, the microbiota contributes to nutrient metabolism and synthesis. "Enterotype" describes the collective functional digestive and nutritive capacity of the enteric microbiota. Enterotypes may be associated with diet, geography, or body habitus. ${ }^{24,40}$ While enterotypes have not yet been fully defined in human infants, there is evidence of a breastfed infant enterotype. In the feces of 8 breastfed infants compared with 10 formula-fed infants, metagenomic analysis suggests an increase in carbohydrate metabolism, amino acid and nitrogen metabolism, and cobalamin synthesis. ${ }^{6}$ Similarly, the breast milk metagenome is enriched for nitrogen metabolism, membrane transport, and the oxidative stress response. ${ }^{41}$ In premature and term infants fed breast milk and formula, metagenomic analysis of stool samples has revealed an enhanced virulence potential with the presence of bacteriophage and genes encoding for type III and IV secretion systems. ${ }^{42-44}$ These data are corroborated with an animal model in which there is increased oxidative stress and a decreased production of proteins utilized in cell adhesion with formula feedings compared with breast milk feedings. ${ }^{45}$

The breast milk microbiota also may be involved in enhancing intestinal barrier protection. Animal studies have demonstrated that enteric colonization is critical for upregulation of epithelial junctional complexes, stimulation of antimicrobial peptide defenses, and expression of key detoxifying enzymes such as alkaline phosphatase to mitigate overstimulation by bacterial lipopolysaccharide ligands. ${ }^{46-48}$ In an animal model, heat shock protein 70 (HSP70) in breast milk decreased bacterial translocation from the gut lumen. ${ }^{49}$ It is possible that microbiota in breast milk may increase HSP70 levels in the intestinal lumen and contribute to epithelial barrier function in neonates. ${ }^{50}$

Oligosaccharides in breast milk have a dynamic relationship with microbiota in breast milk and the enteric tract. Structurally, these are complex glycans found in human breast milk. Traditionally, oligosaccharides were thought to serve as a substrate for the growth of intestinal bacteria in the distal enteric tract. ${ }^{51}$ Recent data suggest a more complex relationship, through which oligosaccharides in breast milk are not consumed by microbiota but still alter the growth of microbiota. $^{52}$ In a rat model, oligosaccharide levels were diminished in the small intestine and differentially secreted into urine, suggesting selective absorption of oligosaccharides possibly in concert with differing microbiota throughout the enteric tract. ${ }^{53}$ In addition, oligosaccharides have independent immune function in neonates. ${ }^{54}$ Ultimately, oligosaccharides may work synergistically with breast milk and enteric microbiota to strengthen barrier function.

In colonizing or transiting the infant enteric tract, the breast milk microbiota may have broader developmental consequences for the infant. Microbiota in breast milk may also establish a normal gut-brain axis. Animals raised in GF environments have decreased intestinal peristalsis that can be restored with the introduction of enteric microbiota from animals with conventional microbial exposure. ${ }^{55,56}$ In a comparison of GF, specific pathogen-free (SPF), and gnotobiotic animals, GF showed an exaggerated stress response compared with SPF mice. This response could be reversed with early exposure to Bifidobacterium infantis, an organism that has been identified in breast milk. ${ }^{14,57}$ Oral antibiotic administration to animals raised in an SPF environment alters enteric microbiota, upregulates brain-derived neurotropic factor, and increases exploratory behavior. ${ }^{58}$ Further work will elucidate the relationship between breast milk microbiota, the developing enteric microbiota, and the gut-brain axis. 


\section{Mastitis: A Case of Dysbiosis of Breast Milk Microbiota}

Mastitis is defined as inflammation of the breast, with or without infection. ${ }^{59}$ Although Staphylococcus aureus has traditionally been considered the primary cause of infectious mastitis, in recent studies, in the breast milk of healthy women. Kvist et $\mathrm{al}^{60}$ compared bacterial species in milk samples from 192 women with a clinical diagnosis of mastitis versus 466 healthy controls. S. aureus was present in $45 \%$ of women with mastitis and $31 \%$ of healthy donors, and mean colony counts were identical in the two groups. Moreover, the authors found no correlation between colony counts and symptom severity among women with mastitis. These results suggest the presence of $S$. aureus in breast milk does not independently result in clinical mastitis.

Delgado et $\mathrm{al}^{61}$ have explored the role of coagulase-negative Staphylococcus spp. in mastitis. Using pulsed field gel electrophoresis genotyping to identify species present in the milk of women with mastitis symptoms, they found that S. epidermidis was present in $85 \%$ (17/20) of samples, compared with S. aureus in $40 \%$ (8/20) of samples. ${ }^{61}$ Milk samples were collected after cleansing the nipple and areola with soap and sterile water, before applying chlorhexidine, to minimize contamination with skin microbiota. They subsequently compared strains of $S$. epidermidis present in women with mastitis versus healthy controls. Women with clinical signs of infection were more likely to harbor strains of S. epidermidis with the $i c a D$ gene (33 vs. $11 \%, p=0.03$ ), which was correlated with biofilm production. ${ }^{62}$ Thus, virulence factors of $S$. epidermidis strains found in breast milk may contribute to the pathogenesis of mastitis.

Based on tentative evidence from animal studies and clinical trials, probiotic-like organisms may compete with putative mastitis pathogens, and administration of probiotics may prevent or ameliorate mastitis symptoms. In an in vitro bovine animal model, certain strains of Lactobacillus inhibited adhesion and internalization of Staphylococcus spp. by mammary epithelial cells. ${ }^{63}$ Lactobacilli also produce anti-inflammatory and antimicrobial factors. ${ }^{64}$ In clinical trials, oral administration of probiotics to women with mastitis appears to reduce mastitis symptoms, and orally administered strains can be detected in human milk. ${ }^{65}$ Arroyo et al tested human milk-derived probiotic strains for treatment of mastitis in 352 women. ${ }^{66}$ They reported a greater reduction in bacterial counts of $S$. epidermidis, S. aureus, and S. mitis, as well as greater reduction in pain, in the probiotic-treated group, compared with antibiotictreated controls.

Of note, the methods reported in the published manuscript differ from ClinicalTrials.gov (NCT00716183) in several ways, including the types of probiotics and antibiotics used and the study allocation method, which is described on Clinical Trials as an open-label, nonrandomized trial and as a randomized, double-blinded trial in the published manuscript. Collectively, based on these results, clinical mastitis may result from virulent $S$. epidermidis strains in breast milk and may be moderated with probiotic therapy.

\section{Conclusion}

In summary, breast milk has a dynamic microbial ecology with a microbiota composed of skin- and enteric-associated bacteria and pathogenic viruses. These organisms are transferred from maternal and infant microbial communities into breast milk via multiple mechanisms, including transmission from skin, movement from infant oral cavity into the mammary gland during breastfeeding, EMT, and reactivation from mammary gland in the case of CMV and HIV. Microbiota in breast milk advance neonatal immune function, enhance nutrient metabolism, improve intestinal barrier function, and contribute to the development of the gut-brain neural axis. The pathogenesis of clinical mastitis may result from dysbiosis in the mammary gland with virulent strains of Staphylococcus spp. Administration of probiotics may reduce the symptoms of mastitis by restoring mammary gland and breast milk microbiota. With improved understanding of the impact of breast milk microbiota, it may be possible to manipulate these microbial communities to improve the health and development of mothers and their neonates.

\section{References}

1 Han YW, Shen T, Chung P, Buhimschi IA, Buhimschi CS. Uncultivated bacteria as etiologic agents of intra-amniotic inflammation leading to preterm birth. J Clin Microbiol 2009;47(1):38-47

2 D'Onofrio A, Crawford JM, Stewart EJ, et al. Siderophores from neighboring organisms promote the growth of uncultured bacteria. Chem Biol 2010;17(3):254-264

3 Costello EK, Stagaman K, Dethlefsen L, Bohannan BJ, Relman DA. The application of ecological theory toward an understanding of the human microbiome. Science 2012;336(6086):1255-1262

4 Grice EA, Kong HH, Conlan S, et al; NISC Comparative Sequencing Program. Topographical and temporal diversity of the human skin microbiome. Science 2009;324(5931):1190-1192

5 Gao Z, Tseng CH, Pei Z, Blaser MJ. Molecular analysis of human forearm superficial skin bacterial biota. Proc Natl Acad Sci U S A 2007;104(8):2927-2932

6 Yatsunenko T, Rey FE, Manary MJ, et al. Human gut microbiome viewed across age and geography. Nature 2012;486(7402): 222-227

7 Gueimonde M, Laitinen K, Salminen S, Isolauri E. Breast milk: a source of bifidobacteria for infant gut development and maturation? Neonatology 2007;92(1):64-66

8 Heikkilä MP, Saris PE. Inhibition of Staphylococcus aureus by the commensal bacteria of human milk. J Appl Microbiol 2003;95(3): 471-478

9 Tyson JE, Edwards WH, Rosenfeld AM, Beer AE. Collection methods and contamination of bank milk. Arch Dis Child 1982;57(5): 396-398

10 Hunt KM, Foster JA, Forney LJ, et al. Characterization of the diversity and temporal stability of bacterial communities in human milk. PLoS ONE 2011;6(6):e21313

11 Cabrera-Rubio R, Collado MC, Laitinen K, Salminen S, Isolauri E, Mira A. The human milk microbiome changes over lactation and is shaped by maternal weight and mode of delivery. Am J Clin Nutr 2012;96(3):544-551

12 Thompson N, Pickler RH, Munro C, Shotwell J. Contamination in expressed breast milk following breast cleansing. J Hum Lact 1997; 13(2):127-130 
13 Palmer C, Bik EM, DiGiulio DB, Relman DA, Brown PO. Development of the human infant intestinal microbiota. PLoS Biol 2007; 5(7):e177

14 Perez PF, Doré J, Leclerc M, et al. Bacterial imprinting of the neonatal immune system: lessons from maternal cells? Pediatrics 2007;119(3):e724-e732

15 Holmes AV. Establishing successful breastfeeding in the newborn period. Pediatr Clin North Am 2013;60(1):147-168

16 Ramsay DT, Kent JC, Owens RA, Hartmann PE. Ultrasound imaging of milk ejection in the breast of lactating women. Pediatrics 2004; 113(2):361-367

17 Ramsay DT, Mitoulas LR, Kent JC, Larsson M, Hartmann PE. The use of ultrasound to characterize milk ejection in women using an electric breast pump. J Hum Lact 2005;21(4):421-428

18 Lif Holgerson P, Harnevik L, Hernell O, Tanner AC, Johansson I. Mode of birth delivery affects oral microbiota in infants. J Dent Res 2011;90(10):1183-1188

19 Stagg AJ, Hart AL, Knight SC, Kamm MA. The dendritic cell: its role in intestinal inflammation and relationship with gut bacteria. Gut 2003;52(10):1522-1529

20 Donnet-Hughes A, Perez PF, Doré J, et al. Potential role of the intestinal microbiota of the mother in neonatal immune education. Proc Nutr Soc 2010;69(3):407-415

21 Turroni F, Peano C, Pass DA, et al. Diversity of bifidobacteria within the infant gut microbiota. PLoS ONE 2012;7(5):e36957

22 Heath L, Conway S, Jones L, et al. Restriction of HIV-1 genotypes in breast milk does not account for the population transmission genetic bottleneck that occurs following transmission. PLoS ONE 2010;5(4):e10213

23 Gray RR, Salemi M, Lowe A, et al. Multiple independent lineages of HIV-1 persist in breast milk and plasma. AIDS 2011;25(2): 143-152

$24 \mathrm{Wu}$ GD, Chen J, Hoffmann C, et al. Linking long-term dietary patterns with gut microbial enterotypes. Science 2011; 334(6052):105-108

25 Wu CA, Paveglio SA, Lingenheld EG, Zhu L, Lefrançois L, Puddington L. Transmission of murine cytomegalovirus in breast milk: a model of natural infection in neonates. J Virol 2011;85(10):5115-5124

26 Vochem M, Hamprecht K, Jahn G, Speer CP. Transmission of cytomegalovirus to preterm infants through breast milk. Pediatr Infect Dis J 1998;17(1):53-58

27 Stagno S, Reynolds DW, Pass RF, Alford CA. Breast milk and the risk of cytomegalovirus infection. N Engl J Med 1980;302(19): 1073-1076

28 Lanzieri TM, Dollard SC, Josephson CD, Schmid DS, Bialek SR. Breast milk-acquired cytomegalovirus infection and disease in VLBW and premature infants. Pediatrics 2013;131(6):e1937-e1945

29 Ehlinger EP, Webster EM, Kang HH, et al. Maternal cytomegalovirus-specific immune responses and symptomatic postnatal cytomegalovirus transmission in very low-birth-weight preterm infants. J Infect Dis 2011;204(11):1672-1682

30 Godfrey WR, Spoden DJ, Ge YG, et al. Cord blood CD4(+)CD25 (+)-derived $\mathrm{T}$ regulatory cell lines express FoxP3 protein and manifest potent suppressor function. Blood 2005;105(2):750-758

31 Zhou L, Chong MM, Littman DR. Plasticity of CD4+ T cell lineage differentiation. Immunity 2009;30(5):646-655

32 Zhang B, Ohtsuka Y, Fujii T, et al. Immunological development of preterm infants in early infancy. Clin Exp Immunol 2005;140(1): 92-96

33 Stephens S, Brenner MK, Duffy SW, Lakhani PK, Kennedy CR, Farrant J. The effect of breast-feeding on proliferation by infant lymphocytes in vitro. Pediatr Res 1986;20(3):227-231

34 Carver JD, Pimentel B, Wiener DA, Lowell NE, Barness LA. Infant feeding effects on flow cytometric analysis of blood. J Clin Lab Anal 1991;5(1):54-56

35 Tarcan A, Gürakan B, Tiker F, Ozbek N. Influence of feeding formula and breast milk fortifier on lymphocyte subsets in very low birth weight premature newborns. Biol Neonate 2004;86(1):22-28
36 Donnet-Hughes A. Protective properties of human milk. In: Duggan D, ed. Nutrition in Pediatrics. 4th ed. Ontario, Canada: Decker Publishing; 2008:355-362

37 Spörri R, Reis e Sousa C. Inflammatory mediators are insufficient for full dendritic cell activation and promote expansion of CD4+ T cell populations lacking helper function. Nat Immunol 2005;6(2): 163-170

38 Hansen $\mathrm{CH}$, Nielsen DS, Kverka M, et al. Patterns of early gut colonization shape future immune responses of the host. PLOS ONE 2012;7(3):e34043

39 M'Rabet L, Vos AP, Boehm G, Garssen J. Breast-feeding and its role in early development of the immune system in infants: consequences for health later in life. J Nutr 2008;138(9):1782S-1790S

40 Arumugam M, Raes J, Pelletier E, et al; MetaHIT Consortium. Enterotypes of the human gut microbiome. Nature 2011; 473(7346):174-180

41 Ward TL, Hosid S, Ioshikhes I, Altosaar I. Human milk metagenome: a functional capacity analysis. BMC Microbiol 2013;13:116

42 Morowitz MJ, Denef VJ, Costello EK, et al. Strain-resolved community genomic analysis of gut microbial colonization in a premature infant. Proc Natl Acad Sci U S A 2011;108(3):1128-1133

43 LaTuga MS, Ellis JC, Cotton CM, et al. Beyond bacteria: a study of the enteric microbial consortium in extremely low birth weight infants. PLoS ONE 2011;6(12):e27858

44 Schwartz S, Friedberg I, Ivanov IV, et al. A metagenomic study of diet-dependent interaction between gut microbiota and host in infants reveals differences in immune response. Genome Biol 2012;13(4):r32

45 Carlisle EM, Poroyko V, Caplan MS, Alverdy J, Morowitz MJ, Liu D. Murine gut microbiota and transcriptome are diet dependent. Ann Surg 2013;257(2):287-294

46 Hancock RE, Scott MG. The role of antimicrobial peptides in animal defenses. Proc Natl Acad Sci U S A 2000;97(16):8856-8861

47 Berkes J, Viswanathan VK, Savkovic SD, Hecht G. Intestinal epithelial responses to enteric pathogens: effects on the tight junction barrier, ion transport, and inflammation. Gut 2003;52(3):439-451

48 Bates JM, Akerlund J, Mittge E, Guillemin K. Intestinal alkaline phosphatase detoxifies lipopolysaccharide and prevents inflammation in Zebrafish in response to the gut microbiota. Cell Host Microbe 2007;2(6):371-382

49 Liedel JL, Guo Y, Yu Y, et al. Mother's milk-induced Hsp70 expression preserves intestinal epithelial barrier function in an immature rat pup model. Pediatr Res 2011;69(5, Pt 1):395-400

50 Arvans DL, Vavricka SR, Ren $\mathrm{H}$, et al. Luminal bacterial flora determines physiological expression of intestinal epithelial cytoprotective heat shock proteins 25 and 72 . Am J Physiol Gastrointest Liver Physiol 2005;288(4):G696-G704

51 Marcobal A, Barboza M, Sonnenburg ED, et al. Bacteroides in the infant gut consume milk oligosaccharides via mucus-utilization pathways. Cell Host Microbe 2011;10(5):507-514

52 Hunt KM, Preuss J, Nissan C, et al. Human milk oligosaccharides promote the growth of staphylococci. Appl Environ Microbiol 2012;78(14):4763-4770

53 Jantscher-Krenn E, Marx C, Bode L. Human milk oligosaccharides are differentially metabolised in neonatal rats. Br J Nutr 2013; 110(4):640-650

54 Eiwegger T, Stahl B, Haidl P, et al. Prebiotic oligosaccharides: in vitro evidence for gastrointestinal epithelial transfer and immunomodulatory properties. Pediatr Allergy Immunol 2010;21(8): $1179-1188$

55 Husebye E, Hellström PM, Midtvedt T. Intestinal microflora stimulates myoelectric activity of rat small intestine by promoting cyclic initiation and aboral propagation of migrating myoelectric complex. Dig Dis Sci 1994;39(5):946-956

56 Husebye E, Hellström PM, Sundler F, Chen J, Midtvedt T. Influence of microbial species on small intestinal myoelectric activity and transit in germ-free rats. Am J Physiol Gastrointest Liver Physiol 2001;280(3):G368-G380 
57 Sudo N, Chida Y, Aiba Y, et al. Postnatal microbial colonization programs the hypothalamic-pituitary-adrenal system for stress response in mice. J Physiol 2004;558(Pt 1):263-275

58 Bercik P, Denou E, Collins J, et al. The intestinal microbiota affect central levels of brain-derived neurotropic factor and behavior in mice. Gastroenterology 2011;141(2):599-609, e1-e3

59 World Health Organization. Mastitis: Causes and Management. Geneva: World Health Organization; 2000

60 Kvist LJ, Larsson BW, Hall-Lord ML, Steen A, Schalén C. The role of bacteria in lactational mastitis and some considerations of the use of antibiotic treatment. Int Breastfeed J 2008;3:6

61 Delgado S, Arroyo R, Martín R, Rodríguez JM. PCR-DGGE assessment of the bacterial diversity of breast milk in women with lactational infectious mastitis. BMC Infect Dis 2008;8:51

62 Delgado S, Arroyo R, Jiménez E, et al. Staphylococcus epidermidis strains isolated from breast milk of women suffering infectious mastitis: potential virulence traits and resistance to antibiotics. BMC Microbiol 2009;9:82

63 Bouchard DS, Rault L, Berkova N, Le Loir Y, Even S. Inhibition of Staphylococcus aureus invasion into bovine mammary epithelial cells by contact with live Lactobacillus casei. Appl Environ Microbiol 2013;79(3):877-885

64 Jones SE, Versalovic J. Probiotic Lactobacillus reuteri biofilms produce antimicrobial and anti-inflammatory factors. BMC Microbiol 2009;9:35

65 Jiménez E, Fernández L, Maldonado A, et al. Oral administration of Lactobacillus strains isolated from breast milk as an alternative for the treatment of infectious mastitis during lactation. Appl Environ Microbiol 2008;74(15):4650-4655

66 Arroyo R, Martín V, Maldonado A, Jiménez E, Fernández L, Rodríguez JM. Treatment of infectious mastitis during lactation: antibiotics versus oral administration of Lactobacilli isolated from breast milk. Clin Infect Dis 2010;50(12):1551-1558 